\title{
Prenatal diagnosis of fetal anomalies from the third to the first trimester and back. (BJOG SInce 1902 on BJOG-20-0525.R1)
}

\author{
Eric Jauniaux ${ }^{1}$ and Federico Prefumo (Italy) ${ }^{2}$ \\ ${ }^{1}$ University College London \\ ${ }^{2}$ University of Brescia
}

July 29, 2020

The prenatal diagnosis of fetal anomalies started with the development of X-ray. In 1943, Hartley and Burnet, Radiologists in Manchester ( $\mathrm{J}$ Obstet Gynaecol Brit Empire,1943;50:1-12), reported a series of 11 cases of "croaniolacunia" or lacunar skull, a condition often associated at births with spina bifida or encephalocele. These cases were all diagnosed in the third trimester of pregnancy and the radiograph features believed to be due to the effect of increased intracranial pressure on the fetal skull of hydrocephaly. Until the end of the 1960s, radiography remained the main technique to diagnose congenital abnormalities. In 1969, Russell (J Obstet Gynaecol Br Commonw,1969;76:345-50), also a consultant radiologist from Manchester, compared the accuracy of antenatal radiology examinations with paediatric reports in the diagnosis of anencephaly and other major neural tube defects, skeletal abnormalities such as achondroplasia and severe exomphalos when associated with rib deformities. Overall, the accuracy of the radiological diagnosis was considered as "strikingly" accurate for neural tube defects with 88 out of 113 cases of anencephaly diagnosed before delivery. Although, the author did not provide the gestational age at diagnosis, the images included in the article indicate that these were obtained in the third trimester. As neonatal care and surgery were in their infancy at the time, the main objective in diagnosing these anomalies was not the fetus but the need to identify antenatally mothers at risk of obstructed labour.

Not surprisingly, some of the first publications by the team of Ian Donald in Glasgow were on the antenatal use of ultrasound imaging in the evaluation of the size of the fetal head (Willocks et al., J Obstet Gynaecol Br Commonw,1964;71:11-20). The fetal head was the only structure that could be measured and biparietal diameter the only measurement that could be obtained with the "ultrasound beam" of the first ultrasound machine (Figure). The technique called "cephalometry" was used at the end of the third trimester to assess "growth and maturity" of the fetus and "disproportion" and was found to be more reliable with ultrasound than X-ray. It would be another decade, before ultrasound imaging could reliably identify fetal anomalies such as spina-bifida in the second trimester of pregnancy (Campbell et al., Lancet,1975;1(7920):1336-7). However, the use of ultrasound imaging in the mid-seventies to search for major neural tube defects was always triggered by high levels of maternal serum alpha-fetoprotein. As there were few ultrasound equipment available and few trained operators, this biomarker was to remain for two decades the first line of action in the antenatal screening strategy for spina-bifida. The advent of high-resolution imaging, access lowcost and mobile ultrasound equipment and the training of more specialists and sonographers has moved the antenatal screening and diagnosis of many fetal anomalies to 11-14 weeks of gestation (Ushakov et al., UOG,2019;54:740-5).

The systematic review by Drukker et al. (BJOG 2020) brings the focus back to late pregnancy: even in our exciting modern era of early anomaly scanning, a fetal abnormality will still be found in about 1 in 300 women scanned in the third trimester.

Word count: 500 
BJOG since 1902 Perspectives on BJOG-20-0525R1

\section{Disclosure of interests}

The authors declare no conflicts of interest.

Eric Jauniaux, Professor, EGA Institute for Women's Health, Faculty of Population Health Sciences, University College London, UK.

Federico Prefumo, Associate Professor, Department of Clinical and Experimental Sciences, University of Brescia, Italy.

\section{BJOG Scientific Editors}

Figure. The apparatus used is a modified commercial ultrasonic flaw detector (the Kelvin Hughes Mark IV), with "A-scope" presentation (from Willocks et al., J Obstet Gynaecol Br Commonw,1964;71:11-20).

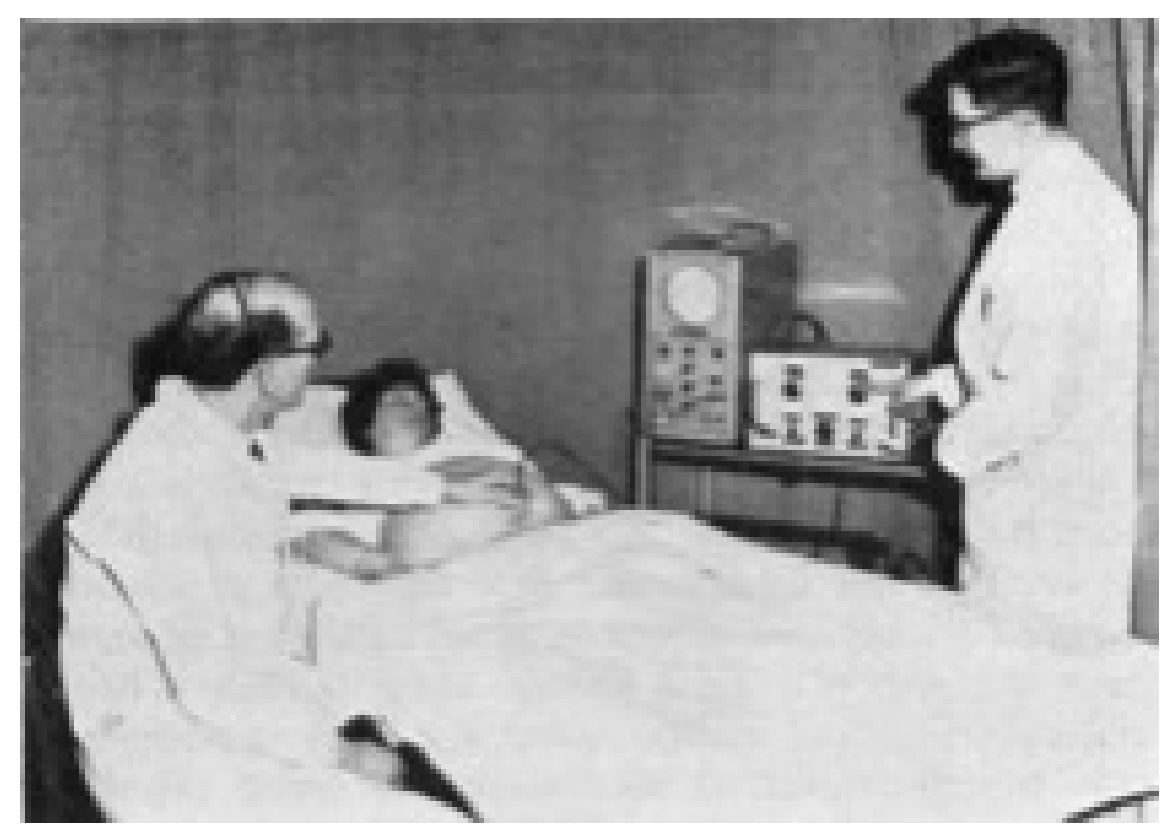

